LIVER DISEASE

\title{
MELD scoring system is useful for predicting prognosis in patients with liver cirrhosis and is correlated with residual liver function: a European study
}

\author{
F Botta, E Giannini, P Romagnoli, A Fasoli, F Malfatti, B Chiarbonello, E Testa, D Risso, \\ G Colla, R Testa
}

See end of article for authors' affiliations

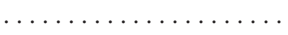

Correspondence to: Professor R Testa,

Gastroenterology Unit, Department of Internal Medicine, University of Genoa, Viale Benedetto XV, No 6, 16132 Genoa, Italy; rtesta@unige.it

Accepted for publication 30 April 2002

\begin{abstract}
Background: Indices for predicting survival are essential for assessing prognosis and assigning priority for liver transplantation in patients with liver cirrhosis. The model for end stage liver disease (MELD) has been proposed as a tool to predict mortality risk in cirrhotic patients. However, this model has not been validated beyond its original setting.

Aim: To evaluate the short and medium term survival prognosis of a European series of cirrhotic patients by means of MELD compared with the Child-Pugh score. We also assessed correlations between the MELD scoring system and the degree of impairment of liver function, as evaluated by the monoethylglycinexylidide (MEGX) test.

Patients and methods: We retrospectively evaluated survival of a cohort of 129 cirrhotic patients with a follow up period of at least one year. The Child-Pugh score was calculated and the MELD score was computed according to the original formula for each patient. All patients had undergone a MEGX test. Multivariate analysis was performed on all variables to identify the parameters independently associated with one year and six month survival. MELD values were correlated with both Child-Pugh scores and MEGX test results.

Results: Thirty one patients died within the first year of follow up. Child-Pugh and MELD scores, and MEGX serum levels were significantly different among patients who survived and those who died. Serum creatinine, international normalised ratio, and MEGX $\mathrm{X}_{60}$ were independently associated with six month mortality while the same variables and the presence of ascites were associated with one year mortality. MELD scores showed significant correlations with both MEGX values and Child-Pugh scores. Conclusions: In a European series of cirrhotic patients the MELD score is an excellent predictor of both short and medium term survival, and performs at least as well as the Child-Pugh score. An increase in MELD score is associated with a decrease in residual liver function.
\end{abstract}

Thar ere is an increasing discrepancy between the number of cirrhotic patients on waiting lists for orthotopic liver transplantation (OLT) and the number of available donor livers. ${ }^{1}$ The correct timing for surgery has an important impact on both mortality and morbidity of the patients. ${ }^{2}$ Indeed, late transplantation affects patient outcome while performing OLT in those with less urgent indications penalises patients who could benefit most from the procedure. ${ }^{3}$ Moreover, the economics, social burden, and ethical considerations of maintaining patients on waiting lists should also be taken into consideration. ${ }^{4}$

Over the years, many clinical and biochemical parameters have been suggested in order to more accurately predict the prognosis of cirrhotic patients and correctly assess their short and medium term survival. The Child-Pugh score is still considered the cornerstone in the prognostic evaluation of cirrhotic patients although it was formulated more than 30 years ago. Nevertheless, it has some drawbacks such as subjectivity of clinical parameters and limited discriminant ability. $^{5-7}$

Child-Pugh class A patients usually show good medium term survival without OLT unless other events (for example, hepatocellular carcinoma, uncontrolled bleeding due to portal hypertension, etc) occur, ${ }^{18}$ while Child-Pugh class $C$ patients are considered the conventional candidates for the procedure. Child-Pugh class B patients can be considered a heterogeneous group as their clinical condition may remain stable for more than a year or rapidly deteriorate. ${ }^{9}$
Quantitative liver function tests that evaluate the clearance of exogenous substances have been studied and combined with the Child-Pugh classification or with other scoring systems to create composite prognostic scores in an attempt to improve prognostic accuracy. ${ }^{50-13}$ The monoethylglycinexylidide (MEGX) test, which depends on liver blood flow and cytochrome P450 activity, is a quantitative liver function test related to the severity of liver disease, ${ }^{14}$ and shows good correlation with medium and short term survival prognosis of cirrhotic patients. ${ }^{6} 15-20$

Recently, the model for end stage liver disease (MELD) was introduced as a tool to predict mortality risk and to assess disease severity in patients with liver cirrhosis so as to determine organ allocation priorities. ${ }^{21}$ Although previoulsy formulated as a prognostic index for cirrhotic patients undergoing transjugular intrahepatic portosystemic stent shunt (TIPSS), it was validated by the same authors on a broad series of patients with liver disease of various aetiology and severity. ${ }^{22}{ }^{23}$ Nevertheless, although the MELD score takes into consideration objective parameters (serum creatinine,

Abbreviations: OLT, orthotopic liver transplantation; MEGX monoethylglycinexylidide; MELD, model for end stage liver disease; TIPSS, transjugular intrahepatic portosystemic stent shunt; INR, international normalised ratio; $\mathrm{HCV}$, hepatitis $C$ virus; $H B V$, hepatitis $B$ virus; HDV, hepatitis D virus; ROC, receiver operating characteristic; SS, sensitivity; $S P$, specificity; $R R$, relative risk. 
Table 1 Clinical and biochemical characteristics of the 129 cirrhotic patients subdivided according to one year survival

\begin{tabular}{|c|c|c|c|}
\hline & Surviving patients & Deceased patients & $\mathrm{p}$ Value \\
\hline $\operatorname{Sex}(M / F)$ & $70 / 28$ & $25 / 6$ & NS \\
\hline Age $(y)$ & $(22-75)$ & (31-71) & NS \\
\hline Encephalopathy (yes/no) & 7/91 & $5 / 26$ & NS \\
\hline Ascites (yes/no) & $32 / 66$ & $21 / 10$ & 0.0007 \\
\hline Bilirubin (mg/dl) & $2(0.3-12)$ & $2.4(0.4-7)$ & NS \\
\hline Albumin (g/dl) & $3.5(2.4-5)$ & $3.4(2.3-5)$ & NS \\
\hline Prothrombin activity (\%) & 59 (31-100) & $52 \quad(30-85)$ & 0.01 \\
\hline Child-Pugh score & $7 \quad(5-12)$ & $9 \quad(5-14)$ & 0.001 \\
\hline Creatinine (mg/dl) & $0.9(0.6-1.3)$ & $1.0(0.7-1.2)$ & NS \\
\hline INR & $1.6(1.0-2.9)$ & $1.8(1.1-2.9)$ & 0.01 \\
\hline MELD score & $6 \quad(-5-17)$ & $9(0-19)$ & 0.003 \\
\hline$M E L D_{\text {aetiology }}$ score & $(-5-23)$ & (0-25) & 0.003 \\
\hline $\operatorname{MEGX}_{15}(\mathrm{ng} / \mathrm{ml})$ & $(0-110)$ & (0-44) & 0.003 \\
\hline MEGX $_{30}(\mathrm{ng} / \mathrm{ml})$ & $(2-91)$ & $(1-63)$ & $<0.001$ \\
\hline $\operatorname{MEGX}_{60}(\mathrm{ng} / \mathrm{ml})$ & (4-93) & $18(1-46)$ & $<0.01$ \\
\hline
\end{tabular}

Data are median (range).

INR, international normalised ratio; MELD, model for end stage liver disease; MELD aetiologe, $_{\text {, }}$ odel for end stage liver disease computed including aetiology of liver disease; MEGX, monoethylglycinexylidide.

the international normalised ratio (INR), bilirubin levels) and is computed with statistically derived coefficients on a continuous scale with no upper or lower limits, thus avoiding many drawbacks of the Child-Pugh score, it has generated some criticism. ${ }^{24} 25$

In this study our aim was to evaluate the short and medium term prognosis of a European series of cirrhotic patients by means of the MELD score compared with the Child-Pugh score. Moreover, we assessed correlations between the MELD score and both the Child-Pugh score and residual liver function, as evaluated by the MEGX test. Lastly, we performed these analyses focusing on Child-Pugh class B patients due to the peculiar importance of the prognostic accuracy in this subgroup of patients.

\section{PATIENTS AND METHODS}

We retrospectively studied 129 cirrhotic patients $(95$ males, 34 females; median age 50 years, range 22-75) consecutively referred to our unit who were eligible for hepatic functional assessment, and with a follow up of at least one year. Patients with hepatorenal syndrome were not eligible, while none of the patients had spontaneous bacterial peritonitis. Patients with hepatocellular carcinoma were excluded from the study. Liver cirrhosis was diagnosed on the basis of histological, clinical, biochemical, and instrumental results. Aetiology of liver disease was hepatitis C virus (HCV) in 60 patients, hepatitis B virus (HBV) in 21 (among these six were positive for hepatitis D virus (HDV)), HCV and HBV in two, HCV and HBV with HDV in one patient, alcohol abuse in 28 , HCV and alcohol abuse in nine, and autoimmune in eight.

The Child-Pugh score was calculated ${ }^{26}$ and patients were classified as follows: 43 class A ( 31 males and 12 females; median age 52 years, range 22-75), 63 class B (45 males and 18 females; median age 48 years, range 28-71), and 23 class C ( 19 males and four females; median age 48 years, range 31-71). The MELD score was calculated according to the original formula proposed by the Mayo Clinic group:

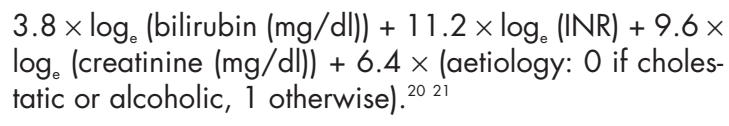

Moreover, we computed the MELD score with or without aetiology of liver disease.
All patients had undergone the MEGX test as follows: lidocaine was injected into a forearm vein over approximately 2-3 minutes at a dose of $1 \mathrm{mg} / \mathrm{kg}$ body weight, and blood samples were then taken 15, 30, and 60 minutes after lidocaine injection $\left(\mathrm{MEGX}_{15}, \mathrm{MEGX}_{30}\right.$, and MEGX $\left.\mathrm{X}_{60}\right)$. MEGX serum concentration was measured at the three sampling times by means of the TDx fluorescence polarisation immunoassay system, ${ }^{27}$ and was calculated as follows: $M E G X_{t}-M{ }^{2} X_{0}$, where $M E G X_{t}$ is MEGX concentration at sampling time and MEGX $\mathrm{X}_{0}$ is MEGX concentration at baseline.

Both Child-Pugh and MELD scores were calculated on parameters obtained at referral. Biochemical evaluations were carried out by the same laboratory. Prothrombin expressed as per cent activity was converted to prothrombin time INR using internal standards of the laboratory and were assessed by a single operator (GC).

Statistical analysis was first performed on the whole group of 129 patients and then on the subgroup of 63 Child-Pugh class B patients, evaluating six month and one year survival. Comparisons between groups were performed using Fisher's exact test and the Mann-Whitney $U$ test. Results are expressed as median (range). The correlation between variables was evaluated by means of Spearman's rank correlation test $\left(r_{s}\right)$. Receiver operating characteristic (ROC) curves were used to determine the cut off values of MEGX serum concentrations, Child-Pugh score, and MELD, with the best sensitivity (SS) and specificity (SP) in discriminating between patients who survived and those who died. The validity of the models was measured by means of the concordance (c) statistic (equivalent to the area under the ROC curve). ${ }^{28} \mathrm{~A} c$ value of $0.8-0.9$ indicates excellent diagnostic accuracy; a model with a $c$ value $>0.7$ should be considered useful. Univariate survival curves were estimated using the Kaplan-Meier method. Stepwise survival analysis was performed by means of Cox regression to determine the variables independently associated with one year and six month mortality. For all analyses a p value $<0.05$ was considered statistically significant. Data were analysed using the SPSS package for Windows (SPSS Inc., Chicago Illinois, USA).

\section{RESULTS}

During the one year follow up, 31 patients died: five were Child-Pugh class A (12\%), 16 were class B (25\%), and 10 were class C $(44 \%)$. The causes of death were all related to liver disease. Ninety eight patients survived more than one year: 38 were Child-Pugh class A (88\%), 47 were class B (75\%), and 13 were class C (56\%). 
Table 2 Clinical and biochemical characteristics of the 129 cirrhotic patients subdivided according to six month survival

\begin{tabular}{|c|c|c|c|}
\hline & Survived patients & Deceased patients & $\mathrm{p}$ Value \\
\hline $\operatorname{Sex}(M / F)$ & $86 / 31$ & $9 / 3$ & NS \\
\hline Age $(y)$ & $49 \quad(22-75)$ & (35-71) & NS \\
\hline Encephalopathy (yes/no) & $10 / 107$ & $1 / 11$ & NS \\
\hline Ascites (yes/no) & $44 / 73$ & $9 / 3$ & 0.03 \\
\hline Bilirubin (mg/dl) & $1.7(0.3-12)$ & $3.1(1.6-6.1)$ & 0.002 \\
\hline Albumin (g/dl) & $3.5(2.4-5)$ & $2.9(2.3-4.3)$ & 0.002 \\
\hline Prothrombin activity (\%) & $58(31-100)$ & $50 \quad(30-85)$ & 0.01 \\
\hline Child-Pugh score & $7 \quad(5-12)$ & $10(6-14)$ & 0.0002 \\
\hline Creatinine (mg/dl) & $0.9(0.6-1.3)$ & $1.0(0.8-1.3)$ & NS \\
\hline INR & $1.6(1.0-2.9)$ & $1.9(1.1-2.9)$ & 0.01 \\
\hline MELD score & $7 \quad(-5-17)$ & $11(6-19)$ & 0.0007 \\
\hline MELD ${ }_{\text {aetiology }}$ score & $12(-5-23)$ & 16 (13-25) & 0.0002 \\
\hline $\operatorname{MEGX}_{15}(\mathrm{ng} / \mathrm{ml})$ & $13.8(0-110.8)$ & $7.2(0-29.7)$ & 0.03 \\
\hline $\mathrm{MEGX}_{30}(\mathrm{ng} / \mathrm{ml})$ & $20.2(2.2-90.9)$ & $9 \quad(0.58-39)$ & 0.0007 \\
\hline $\operatorname{MEGX}_{60}(\mathrm{ng} / \mathrm{ml})$ & 26.1 (4.1-92.9) & $14.3(0.95-40)$ & 0.001 \\
\hline
\end{tabular}

Data are median (range).

INR, international normalised ratio; MELD, model for end stage liver disease; MELD atiologe, $_{\text {, }}$ odel for end

stage liver disease computed including aetiology of liver disease; MEGX, monoethylglycinexylidide.

Tables 1 and 2 show the clinical and biochemical characteristics of the patients, their MELD scores, and their MEGX serum levels 15, 30, and 60 minutes after lidocaine administration, respectively, after both a one year and six month period of follow up. Median Child-Pugh as well as MELD scores and MEGX serum levels at each sampling time were significantly different both at six and 12 months between patients who survived and those who died. Ascites was clinically detected more frequently among deceased patients compared with those who survived.

The cut off values with the best SS and SP in predicting six month and one year survival for Child-Pugh score, MELD scores, and MEGX serum levels were calculated using ROC curves. Moreover, $c$ index was calculated to evaluate the accuracy of the three models. Child-Pugh score, $\mathrm{MEGX}_{30}$, and MEGX $_{60}$ showed the best SS in predicting six month survival while MEGX $_{15}$ had the best SP. Both Child-Pugh and MELD scores had excellent prognostic accuracy (table 3). Moreover, we observed no differences in prognostic accuracy among the various prognostic parameters (comparison of ROC curves). The MELD score showed the best SS in predicting one year survival compared with both the Child-Pugh score and MEGX test, although with low SP (table 4). All variables evaluated showed good one year prognostic accuracy, without significant differences among the various parameters. Figure 1 shows that the Child-Pugh and MELD scores as well as MEGX $_{60}$ cut offs values identified by means of ROC curves clearly differentiated between patients with different survival times.

Table 3 Six month survival sensitivity, specificity, and $c$ index of the Child-Pugh score, MELD scores, and MEGX serum levels at each sampling time

\begin{tabular}{lcccc}
\hline & Cut off & $\begin{array}{l}\text { Sensitivity } \\
(\%)\end{array}$ & $\begin{array}{l}\text { Specificity } \\
(\%)\end{array}$ & c index \\
\hline Child-Pugh score & 8 & 92 & 72 & 0.824 \\
MELD score & 8 & 75 & 68 & 0.796 \\
MELD $_{\text {oeitology }}$ score & 14 & 75 & 72 & 0.823 \\
MEGX $_{15}(\mathrm{ng} / \mathrm{ml})$ & 4 & 50 & 91 & 0.689 \\
MEGX $_{30}(\mathrm{ng} / \mathrm{ml})$ & 15.2 & 92 & 63 & 0.799 \\
MEGX $_{60}(\mathrm{ng} / \mathrm{ml})$ & 22.8 & 92 & 61 & 0.782 \\
\hline
\end{tabular}

MELD, model for end stage liver disease; $M E L D_{\text {aetiolog, }}$ model for end stage liver disease computed including aetiology of liver disease; MEGX, monoethylglycinexylidide.
Multivariate analysis was performed on variables contained in both the Child-Pugh and MELD scores, and on all MEGX sampling times to identify parameters independently associated with six month and one year mortality. This analysis showed that INR (relative risk (RR) 4.282 (range $1.438-12.751$ ); $p=0.009$ ), creatinine serum levels (RR 8.785 (0.213-36.186); $p=0.03)$, and MEGX ${ }_{60}(R R$ $0.920(0.860-0.983) ; p=0.01)$ were the variables significantly associated with six month mortality, while the presence of ascites (RR $2.146(1.203-3.895) ; p=0.01)$, creatinine serum levels (RR 1.473 (1.091-2.014); $\mathrm{p}=0.01$ ), INR (RR 2.47

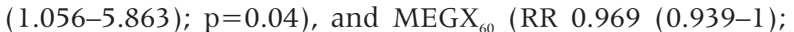
$\mathrm{p}=0.05$ ) were significantly associated with one year mortality.

MELD scores showed a highly significant correlation with both MEGX serum levels $\left(\mathrm{MEGX}_{15}, r_{\mathrm{s}}=-0.545, \mathrm{p}<0.0001\right.$; MEGX $\left._{30}, r_{\mathrm{s}}=-0.617, \mathrm{p}<0.0001 ; \mathrm{MEGX}_{60}, r_{\mathrm{s}}=-0.542, \mathrm{p}<0.0001\right)$ and Child-Pugh scores $\left(r_{\mathrm{s}}=0.817, \mathrm{p}<0.0001\right)$. Moreover, Child-Pugh scores were correlated with MEGX serum levels $\left(\mathrm{MEGX}_{15}, r_{\mathrm{s}}=-0.529, \mathrm{p}<0.0001 ; \mathrm{MEGX}_{30}, r_{\mathrm{s}}=-0.598\right.$, $\left.\mathrm{p}<0.0001 ; \mathrm{MEGX}_{60}, r_{\mathrm{s}}=-0.577, \mathrm{p}<0.0001\right)$.

\section{Prognostic analysis of Child-Pugh class B patients}

Among the various clinical, biochemical, and functional parameters of the 63 Child-Pugh class B patients, only MEGX ${ }_{30}$ $(16.4 \mathrm{ng} / \mathrm{ml}(2.9-63.3) \vee 9.3(0.58-13.3) ; \mathrm{p}=0.02)$ and $\mathrm{MEGX}_{60}$ $(24.6 \mathrm{ng} / \mathrm{ml} \quad(6.7-55.6) \quad v 13.9 \quad(0.9-18.5) ; \mathrm{p}=0.02)$ were significantly lower among patients who died in the short term (six months), while MEGX $_{30}$ (19 ng/ml (4-54) v ll (1-63); $p=0.05)$ was lower among patients who died in the medium term (one year).

Table 4 One year survival sensitivity, specificity, and $c$ index of the Child-Pugh score, MELD scores, and MEGX serum levels at each sampling time

\begin{tabular}{lcccc}
\hline & Cut off & $\begin{array}{l}\text { Sensitivity } \\
(\%)\end{array}$ & $\begin{array}{l}\text { Specificity } \\
(\%)\end{array}$ & c index \\
\hline Child-Pugh score & 8 & 61 & 75 & 0.691 \\
MELD score $_{M^{2}}$ & 6 & 87 & 42 & 0.675 \\
MELD $_{\text {aetiology score }}$ & 12 & 74 & 58 & 0.680 \\
MEGX $_{30}(\mathrm{ng} / \mathrm{ml} / \mathrm{ml})$ & 11 & 74 & 63 & 0.676 \\
MEGX $_{60}(\mathrm{ng} / \mathrm{ml})$ & 18 & 81 & 64 & 0.703 \\
\hline
\end{tabular}

MELD, model for end-stage liver disease; $M E L D_{\text {aetiology }}$ model for end stage liver disease computed including aetiology of liver disease; MEGX, monoethylglycinexylidide. 

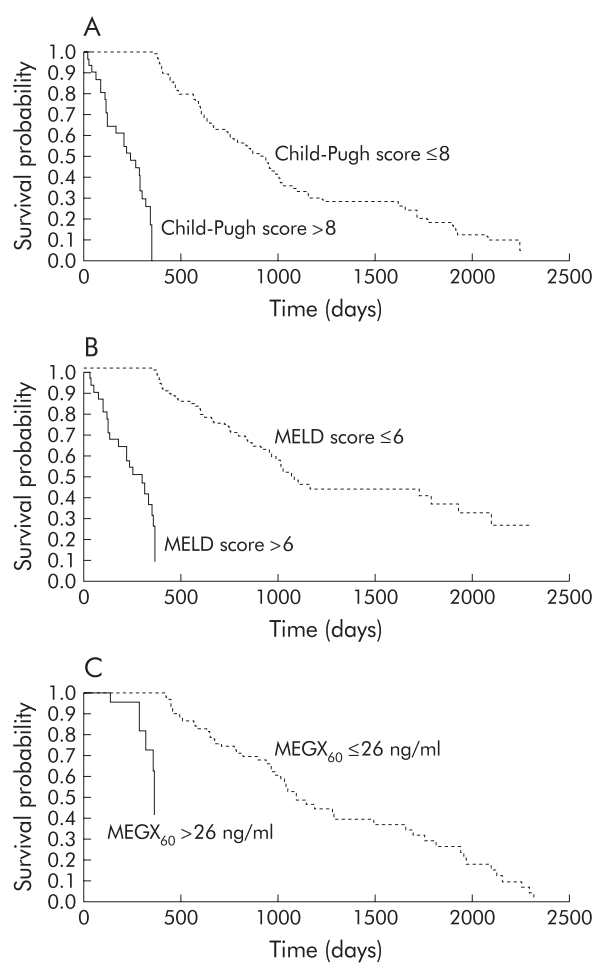

Figure 1 Kaplan-Meier estimated survival curves by (A) Child-Pugh score $(p<0.0001),(B)$ model for end stage liver disease (MELD) score $(p<0.0001)$, and $(C)$ monoethylglycinexylidide concentration at 60 minutes $\left(M E G X_{60}\right)(p<0.0001)$.

Multivariate analysis showed that MEGX $_{60}$ (RR 0.867 $(0.767-0.980) ; p=0.02)$ was independently associated with six month mortality while this analysis was unable to identify any variable associated with one year mortality.

Lastly, correlations between MELD scores and MEGX serum levels $\left(\mathrm{MEGX}_{15}, r_{\mathrm{s}}=-0.356, \mathrm{p}=0.005 ; \mathrm{MEGX}_{30}, r_{\mathrm{s}}=-0.442\right.$, $\mathrm{p}<0.0001$; MEGX60, $\left.r_{\mathrm{s}}=-0.262, \mathrm{p}=0.039\right)$ and Child-Pugh scores $\left(r_{\mathrm{s}}=0.556, \mathrm{p}<0.0001\right)$ were maintained even in this smaller subgroup of patients.

\section{DISCUSSION}

Prognostic evaluation of patients with liver cirrhosis is an important topic often challenging the clinician. The number of patients on waiting lists for OLT is becoming increasingly higher compared with the number of available donor livers. Correct timing of OLT can reduce the mortality of patients on waiting lists and improve post-transplant survival. Moreover, accurate prognosis of patients with cirrhosis is important so as to plan their management as well as the choice of major procedures (for example, TIPSS, surgical, or locoregional treatment for hepatocellular carcinoma).

The Child-Pugh score is an important component of the prognostic evaluation of cirrhotic patients and of the current organ allocation policy, although this traditional score has several shortcomings such as subjectivity of some parameters and limited discriminant ability. In order to overcome the limits of the Child-Pugh score, previous studies have evaluated a "combined score" with quantitative liver function tests, ${ }^{5}$ 10 $^{11} 15$ have created new scores, or have applied scores that were originally formulated to evaluate multiorgan insufficiency in critically ill patients to cirrhotic patients. ${ }^{29-31}$

Recently, the study group at the Mayo Clinic introduced a new scoring system (MELD) to evaluate the prognosis of patients undergoing TIPSS. ${ }^{21}$ They then generalised its application to patients with different stages of liver cirrhosis in order to evaluate their short term survival prognosis. ${ }^{22}{ }^{23}$ Lastly, two independent studies performed in North American cirrhotic patients showed that the MELD score performed at least as well as the Child-Pugh score in predicting patient outcome following acute variceal bleeding $^{32}$ and mortality in patients referred for OLT. ${ }^{33}$ Nevertheless, in Europe the MELD scoring system has been evaluated only in the prognostic assessment of patients undergoing TIPSS. ${ }^{34}$

In this study our aim was to evaluate the short and medium term prognostic ability of the MELD scoring system compared with the Child-Pugh score in a European series of cirrhotic patients who were referred to a tertiary care medical centre and who were followed up for at least one year. Moreover, we assessed the correlations among these staging systems and the degree of liver functional impairment, as evaluated by means of the MEGX test. Lastly, we performed these analyses focusing on Child-Pugh class B patients due to the peculiar importance of the prognostic accuracy in this subgroup of patients.

Firstly, we observed that Child-Pugh and MELD scores, and MEGX could discriminate between deceased and surviving patients both in the short and medium term. Moreover, we showed that these parameters had high diagnostic accuracy for predicting six and 12 month mortality although none performed better than the others. Secondly, we found that among the clinical variables of the traditional score, the presence of ascites seemed to be significantly related to both short and medium term mortality as it was clinically detected more frequently among deceased patients. Lastly, multivariate analysis showed that signs of liver decompensation, such as the presence of ascites, subclinical decrease in renal function, INR, and impairment of liver metabolic activity (MEGX) were independently associated with both one year and six month mortality, although the presence of ascites was not associated with earlier death.

For the first time, we found a significant correlation between MELD score and the degree of metabolic liver functional impairment, as well as confirmation of the relationship between MELD and Child-Pugh score. ${ }^{22} 23$

Child-Pugh class B patients were then analysed separately as this subgroup of patients displays a prognosis that may be difficult to evaluate. Indeed, we found that correlations between MELD and degree of liver functional impairment were maintained even in this subgroup of patients, although only MEGX $_{30}$ values were significantly lower in deceased patients than in survivors. While impaired liver function $\left(\mathrm{MEGX}_{60}\right)$ seemed to be the only parameter independently associated with six month mortality, multivariate analysis was unable to identify any variable associated with one year mortality, thus emphasising the complexity of medium term prognostic assessment of this group of patients.

In this study, we confirmed the prognostic ability and accuracy of the MELD scoring system in a setting which was different from that where the score was formulated (for example, ethnic background, features of disease, etc). In fact, the MELD score showed discriminant ability between patients who survived and those who died both at six and 12 months of follow up. Moreover, it showed excellent diagnostic accuracy in predicting short and medium term survival, at least comparable with the Child-Pugh score. Lastly, for the first time we showed that the MELD scoring system, which is simple, reproducible, and contains variables with important physiopathological connotations, correlated significantly with the degree of liver functional impairment.

Our findings are further strengthened by the results of multivariate analysis. Indeed, multivariate analysis showed that parameters contained in the MELD score (INR and 
serum creatinine levels) and the MEGX test results (MEGX ${ }_{60}$ ) were independently associated with six month mortality, and that signs of liver decompensation such as the presence of ascites was an important parameter predicting one year mortality. These results seem to confirm the complementary role of clinical and functional assessment in the prognostic evaluation of cirrhotic patients. Moreover, they indicate the importance of both Child-Pugh and MELD scoring systems. Lastly, they underscore the importance of some parameters that are pathophysiologically inter-related, and that contribute to worsening of the patient's condition, such as the subclinical decrease in renal function and the presence of ascites. Furthermore, these results are in keeping with those of recent studies showing that the presence of ascites as well as renal function impairment represents one of the most important prognostic variables in cirrhotic patients. ${ }^{35} 36$

The correlation between the MELD scoring system and the degree of liver functional impairment that we observed in the whole group was confirmed even in the subgroup of Child-Pugh class B patients, although in this group the MELD score was unable to discriminate between deceased and surviving patients, either in the short or medium term. It is likely that in this subgroup of patients evaluation of dynamic changes in the MELD score would help identify patients at higher risk of death. ${ }^{37}$ Nevertheless, functional evaluation seemed to be the best tool for predicting one year $\left(\mathrm{MEGX}_{30}\right)$ and six month $\left(\right.$ MEGX $\left._{60}\right)$ mortality in Child-Pugh class $\mathrm{B}$ patients.

The fact that the various analyses identified different MEGX sampling times as variables associated with medium and short term prognosis has a sound physiopharmacological basis. In fact, $\mathrm{MEGX}_{30}$ most likely represents comprehensive functional reserve in cirrhotic patients and reflects the combination of both impaired blood flow and metabolic activity. ${ }^{38}$ MEGX $_{15}$ is predominantly influenced by hepatic blood flow and probably represents a "load stress test" while MEGX $_{60}$ is more an expression of residual metabolic capacity. ${ }^{39}$

In conclusion, in this study we have confirmed the prognostic ability of the MELD scoring system for both short and medium term survival, even in a European series of cirrhotic patients. Moreover, for the first time we have shown that the MELD scoring system is correlated with the degree of liver functional impairment. As both the MELD and Child-Pugh scores showed similar accuracy, we feel that they should be considered complementary tools in evaluating the progressive worsening of the clinical condition of cirrhotic patients. Furthermore, particular attention should be paid to the presence of ascites and creatinine serum levels as clinical signs of derangement of more complex activities and markers of a higher risk of death. Lastly, among Child-Pugh class B patients, evaluation of liver functional reserve has an important role in assessing their short term prognosis.

\section{Authors' affiliations}

F Botta*, E Giannini*, P Romagnoli, A Fasoli, F Malfatti,

B Chiarbonello, E Testa, R Testa, Gastroenterology Unit, Department of Internal Medicine, University of Genoa, Italy

D Risso, Department of Health Science, University of Genoa, Genoa,

Italy

G Colla, Department of Laboratory Medicine, S Martino Hospital, Genoa, Italy

*F Botta and E Giannini contributed equally to this work.

\section{REFERENCES}

1 Lucey MR, Brown KA, Everson GT, et al. Minimal criteria for placement of adults on the liver transplant waiting list. Transplantation 1998;66:956-62.
2 Carithers JL Jr. Liver transplantation. American Association for the study of liver diseases. Liver Transp/ 2000;6:122-35.

3 Freeman R, Edwards E. Liver transplant waiting time does not correlate with waiting list mortality: implications for liver allocation policy. Liver Transp/ 2000;6:543-52.

4 Rufat $\mathbf{P}$, Fourquet $F$, Conti $F$, et al. Costs and outcomes of liver transplantation in adults. Transplantation 1999;68:76-83.

5 Oellerich $\mathbf{M}$, Burdelski $M$, Lautz HU, et al. Assessment of pre-transplant prognosis in patients with cirrhosis. Transplantation 1991;51:801-6.

6 Testa R, Valente U, Risso D, et al. Can the MEGX test and serum bile acids improve the prognostic ability of Child-Pugh's score in liver cirrhosis? Eur J Gastroenterol Hepatol 1999;11:559-63.

7 Shrescha R, McKinley C, Showalter R, et al. Quantitative liver function tests define the functional severity of liver disease in early-stage cirrhosis. Liver Transpl Surg 1997;3:166-73.

8 Keeffe EB. Summary of guidelines on organ allocation and patient listing for liver transplantation. Liver Transp/ Surg 1998;4:S108-14.

9 Oellerich M, Burdelski M, Lautz HU, et al. Predictors of one-year pretransplant survival in patients with cirrhosis. Hepatology 1991;14:1029-34.

10 Arrigoni A, Gindro T, Aimo G, et al. Monoethylglycinexlydide test: a prognostic indicator of survival in cirrhosis. Hepatology 1994;20: 383-7.

11 Adler $M$, Verset D, Bouhdid $H$, et al. Prognostic evaluation of patients with parenchymal cirrhosis. J Hepatol 1997;26:642-9.

12 Jalan R, Hayes PC. Review article: quantitative tests of liver function. Aliment Pharmacol Ther 1995;9:263-70.

13 Gindro T, Arrigoni A, Martinasso G, et al. Monoethyl glycine xylidide (MEGX) test evaluation in primary biliary cirrhosis: comparison with Mayo score. Eur J Gastroenterol Hepatol 1997; 12:1 155-9.

14 Testa R, Caglieris S, Risso D, et al. Monoethylglycinexylidide formation measurement as a hepatic function test to assess severity of chronic liver disease. Am J Gastroenterol 1997;92:2268-73.

15 Shiffman ML, Luketic VA, Sanyal AJ, et al. Hepatic lidocaine metabolism and liver histology in patients with chronic hepatitis and cirrhosis. Hepatology 1994;19:933-40.

16 Oellerich $M$, Hartmann $H$, Ringe $B$, et al. Assessment of prognosis transplant candidates by use of the Pugh-MEGX score. Transpl Proc 1993;25: $1116-19$.

17 Shiffman ML, Luketic VA, Sanyal AJ, et al. Use of hepatic lidocaine metabolism to monitor patients with chronic liver disease. Ther Drug Monit 1996;18:372-7.

18 Shiffman ML, Fisher RA, Sanyal Al, et al. Hepatic lidocaine metabolism and complications of cirrhosis. Implications for assessing patient priority for hepatic transplantation. Transplantation 1993;55:830-5.

19 Ercolani G, Grazi G, Callivà R, et al. The lidocaine (MEGX) test as an index of hepatic function: its clinical usefulness in liver surgery. Surgery 2000;127:464-71.

20 Fabbris $L$, Jemmolo RM, Toffolo $G$, et al. The monoethylglycinexylidide test for grading of liver cirrhosis. Aliment Pharmacol Ther 1999; 13:67-75

21 Malinchoc M, Kamath PS, Gordon FD, et al. A model to predict poor survival in patients undergoing transjugular intrahepatic portosystemic shunts. Hepatology 2000;31:864-71.

22 Kamath PS, Wiesner RH, Malinchoc $M$, et al. A model to predict survival in patients with end-stage liver disease. Hepatology 2001;33:464-70

23 Forman LM, Lucey MR. Predicting the prognosis of chronic liver disease: an evolution from Child to MELD. Hepatology 2001;33:473-5.

24 McCaughan GW, Strasser SI. To MELD or not to MELD? Hepatology $2001 ; 34: 215-16$

25 Wiesner RH, McDiarmid SV, Kamath PS, et al. MELD and PELD: application of survival models to liver allocation. Liver Transpl 2001;7:567-80

26 Pugh RNH, Murray Lyon IM, Dawson JL, et al. Transection of the oesophagus for bleeding oesophageal varices. Br J Surg 1973:60:646-64.

27 Oellerich M, Raude E, Burdelski M, et al. Monoethylglycinexylidide formation kinetics: a novel approach to assessment of liver function. $J$ Clin Chem Biochem 1987:25:845-53.

28 Hanley JA, McNeil BJ. The meaning and use of the area under receiver operating characteristic (ROC) curve. Radiology 1982;143:29-36.

29 Singh N, Gayowski T, Wagener MM, et al. Outcome of patients with cirrhosis requiring intensive care unit support: prospective assessment of predictors of mortality. J Gastroenterol 1998;33:73-9.

30 Muto $\mathbf{P}$, Freeman RB, Haug CE, et al. Liver transplant candidate stratification systems. Implications for third-party payors and organ allocation. Transplantation 1994;57:306-8.

31 Zauner C, Schneeweiss B, Schneider B, et al. Short-term prognosis in critically ill patients with liver cirrhosis: an evaluation of a new scoring system. Eur J Gastroenterol Hepatol 2000;12:517-22.

32 Chalasani N, Kahi CJ, Francois F, et al. Mayo clinic end-stage liver disease model (MELD) for predicting patient outcomes following acute variceal bleeding. Hepatology 2001;34:345A.

33 Abouassi SG, Mihas AA, Williams LM, et al. MELD and CTP scores are equivalent predictors of mortality in cirrhotic veterans referred for orthotopic liver transplantation (OLT). Hepatology 200 1;34:207A 
34 Schepke $M$, Roth F, Brensing KA, et al. MELD-score compared to the Child-Pugh and the Emory-score for the prediction of long-term survival in patients undergoing transjugular intrahepatic portosystemic shunting (TIPS). Hepatology 2001;34:183A.

35 Gonzalez E, Rimola A, Navasa M, et al. Liver transplantation in patients with non-biliary cirrhosis: prognostic value of preoperative factors. $J$ Hepatol 1998;28:320-8.

36 Fernàndez-Esparrach $G$, Sànchez-Fueyo A, Ginès $P$, et al. A prognostic model for predicting survival in cirrhosis with ascites. Hepatol 2001;34:46-52.
37 Yousfi MM, Douglas DD, Harrison E, et al. Model for end-stage liver disease (MELD). Dynamic changes in MELD score is important in predicting mortality for patients awaiting liver transplantation (LTX) Hepatology 2001;34:254A.

38 Bargetzi MJ, Aoyama T, Gonzalez FJ, et al Lidocaine metabolism in human liver microsomes by cytochrome P450IIIA4. Clin Pharmacol Ther 1989;46:521-7.

39 Testa R, Campo N, Caglieris S, et al. Lidocaine elimination and monoethylglycinexylidide formation in patients with chronic hepatitis or cirrhosis. Hepatogastroenterol 1998;45:154-9. 